\title{
Bone Graft Augmentation for Severe Glenoid Bone Loss in Primary Reverse Total Shoulder Arthroplasty
}

\author{
Outcomes and Evaluation of Host Bone Contact by 2D-3D Image \\ Registration
}

\author{
Adam Lorenzetti, MD, Jonathan J. Streit, MD, Andres F. Cabezas, MS, Kaitlyn N. Christmas, BS, Joey LaMartina II, MD, \\ Peter Simon, PhD, and Mark A. Frankle, MD \\ Investigation performed at the Foundation for Orthopaedic Research and Education, Tampa, Florida
}

\begin{abstract}
Background: The treatment of patients with severe glenoid bone loss using reverse total shoulder arthroplasty (RSA) is challenging because of the difficulty in obtaining glenoid fixation. The outcomes following primary RSA with structural bone-grafting for severe glenoid bone loss and the amount of native bone support necessary to achieve clinical improvement are unclear.
\end{abstract}

Methods: We reviewed functional outcomes (American Shoulder and Elbow Surgeons [ASES] score, Simple Shoulder Test [SST], visual analog scale [VAS] for pain and function, patient satisfaction, and range of motion) for 57 patients who were treated with a primary RSA and glenoid bone-grafting for severe glenoid bone loss. Three glenoids were classified as type A2; 2, as type B2; and 2, as type C, according to the Walch classification; 16 glenoids, as grade E1; and 19, as grade E3, according to the Sirveaux classification; 9 glenoids, as grade 3, according to the Levigne classification; and 6 were unable to be classified. For the 44 patients with adequate preoperative computed tomographic (CT) data and postoperative radiographs, we evaluated native bone contact under the glenoid baseplate by matching the projected shape of the implant and scapula from the postoperative radiographs with a generated 3-dimensional (3D) model of the preoperative scapula. We then analyzed functional outcomes in relation to native bone support of the baseplate.

Results: At a mean of 46 months (minimum, 24 months), the patients demonstrated significant improvements in function, motion, and pain (change in the ASES total score $=38.6$, change in SST $=5.4$, change in forward elevation $=$ $72.4^{\circ}$, change in abduction $=67.7^{\circ}$, change in external rotation $=24.3^{\circ}$, and change in VAS pain score $=-4.6 ; p<0.001$ for all). On the basis of the generated 3D model, the baseplate contact to host bone was a mean (and standard deviation) of $17 \% \pm 12 \%$ (range, $0 \%$ to $50 \%$ ). There was no significant correlation between host bone coverage and change in the ASES score $(p=0.51)$ for the 44 patients included in this analysis. There were 4 major complications $(7 \%)$ in the study group but no glenoid baseplate failures.

Conclusions: Glenoid bone-grafting in a primary RSA in a shoulder with severe bone loss produces good functional outcomes that do not correlate with the degree of native bone contact under the baseplate. We had observed no glenoid component failures at the time of writing.

Level of Evidence: Therapeutic Level IV. See Instructions for Authors for a complete description of levels of evidence.

\footnotetext{
Disclosure: DJO Surgical provided funding for this study to the Foundation for Orthopaedic Research and Education (FORE). DJO Surgical did not have input into the design, data collection, analysis, or manuscript preparation. One author (M.A.F.) receives royalties and consulting fees from DJO Surgical. On the Disclosure of Potential Conflicts of Interest forms, which are provided with the online version of the article, one or more of the authors checked "yes" to indicate that the author had a relevant financial relationship in the biomedical arena outside the submitted work and "yes" to indicate that the author had a patent issued for the device that is the subject of the article (http://links.Iww.com/JBJSOA/A15).
}

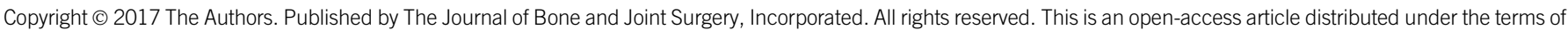

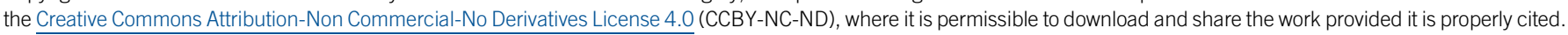
The work cannot be changed in any way or used commercially without permission from the journal. 
$\mathrm{R}$ everse shoulder arthroplasty (RSA) has been established as an effective primary and salvage treatment option for complex disorders of the shoulder that had previously been extremely difficult to treat ${ }^{1-4}$. RSA was initially utilized to treat rotator cuff tear arthropathy and unreconstructible massive rotator cuff tears. Secure fixation to the scapula without cement has been of interest to surgeons faced with severe glenoid bone deficiency. If the amount of native bone available for contact between the scapula and the underside of the baseplate is inadequate, allograft or autograft bone must be used to provide structural support and, if necessary, assist in lateralization of the baseplate. The results of bone-grafting for focal glenoid deficiency in anatomic total shoulder arthroplasty (TSA) have been poor because of graft subsidence and resorption $^{5,6}$. The results of RSA when performed as a revision surgery, sometimes with the use of bone graft, are known to be inferior to those of primary RSA ${ }^{7-14}$. However, little is known about the results of primary RSA when bone graft is utilized for severe focal glenoid deficiency.

We performed a retrospective review of the functional outcomes for our patients following primary RSA with utilization of allograft or autograft bone to supplement a deficient glenoid. Additionally, the degree of bone loss was evaluated using a 2-dimensional/3-dimensional (2D-3D) image registration of preoperative computed tomography (CT) scans and postoperative radiographs. We hypothesized that outcomes of RSA would be positively correlated with the amount of native

TABLE I Demographics of Study Group

\begin{tabular}{|lc|}
\hline \multicolumn{1}{|c}{ Parameter } & Study Group (N =57) \\
\hline Age* (yr) & $73 \pm 8$ \\
Sex (no. [\%]) & $40(70)$ \\
Female & $17(30)$ \\
Male & \\
Side (no. [\%]) & $27(47)$ \\
Left & $30(53)$ \\
Right & \\
Diagnosis (no. [\%]) & $3(5)$ \\
Chronic dislocation & $7(12)$ \\
Osteoarthritis & $38(67)$ \\
Rotator cuff arthropathy & $9(16)$ \\
Rheumatoid arthritis & \\
Preoperative scores* & $17 \pm 10$ \\
ASES function & $20 \pm 12$ \\
ASES pain & $37 \pm 17$ \\
ASES total & $70^{\circ} \pm 34^{\circ}$ \\
Forward elevation & $65^{\circ} \pm 32^{\circ}$ \\
Abduction & $29^{\circ} \pm 24^{\circ}$ \\
External rotation &
\end{tabular}

*The values are given as the mean and the standard deviation.

\begin{tabular}{|lc|}
\multicolumn{1}{c|}{ TABLE II Study Group Rotator Cuff Status and Bone Loss Grading } \\
\hline Parameter & Study Group (N=57) \\
\hline Rotator cuff status - intact (no. [\%]) & $19(33)$ \\
Bone loss (no. [\%]) & \\
Walch type A2 & $3(5)$ \\
Walch type B2 & $2(4)$ \\
Walch type C & $2(4)$ \\
Sirveaux grade E1 & $16(28)$ \\
Sirveaux grade E3 & $19(33)$ \\
Levigne grade 3 & $9(16)$ \\
Unclassifiable & $6(11)$ \\
\hline
\end{tabular}

bone support under the glenoid baseplate at the time of implantation.

\section{Materials and Methods}

Study Subjects

This study was determined to be exempt from review by the 1 Western Institutional Review Board. We identified all patients who had undergone primary RSA with the use of bone-grafting for glenoid deficiency between January 2004 and August 2012. A group of 38 patients (40 shoulders) with $<2$ years of follow-up was excluded. The study group consisted of 57 consecutive patients (mean age and standard deviation, $73 \pm$ 8 years) with minimum 2-year outcome data (mean, 46 months; range, 24 to 105 months) who underwent primary RSA in the setting of substantial glenoid bone loss (Table I). Chart review was performed to identify the diagnosis and surgical indication for each patient. Surgery was performed for an underlying diagnosis of rotator cuff tear arthropathy in 38 shoulders $(67 \%)$, rheumatoid arthritis in 9 shoulders $(16 \%)$, osteoarthritis in 7 shoulders (12\%), and chronic dislocation in 3 shoulders (5\%). In 19 shoulders (33\%), the rotator cuff was intact. Patients demonstrated severe glenoid bone loss, which was identified using the preoperative CT scan to apply the most suitable classification system, on the basis of the underlying diagnosis and bone loss pattern. Sixteen glenoids were classified as grade E1 and 19 glenoids, as grade E3, according to the Sirveaux classification ${ }^{15} ; 9$ glenoids, as grade 3 , according to the Levigne classification ${ }^{3} ; 3$ glenoids, as type $\mathrm{A} 2,2$, as type $\mathrm{B} 2$, and 2 , as type $\mathrm{C}$, according to the Walch classification ${ }^{16}$; and 6 glenoids with severe bone loss were unable to be classified using published classification criteria (Table II).

\section{Clinical Outcomes}

Patients had follow-up evaluations at 1 week, 6 weeks, 3 months, 6 months, 1 year, and annually thereafter, and the American Shoulder and Elbow Surgeons (ASES) score, Simple Shoulder Test (SST), 10-point visual analog scale (VAS) scores for pain and function, and patient satisfaction rating were collected and assessed at a minimum of 2 years (mean, 46 months) from the date of surgery for comparison with 


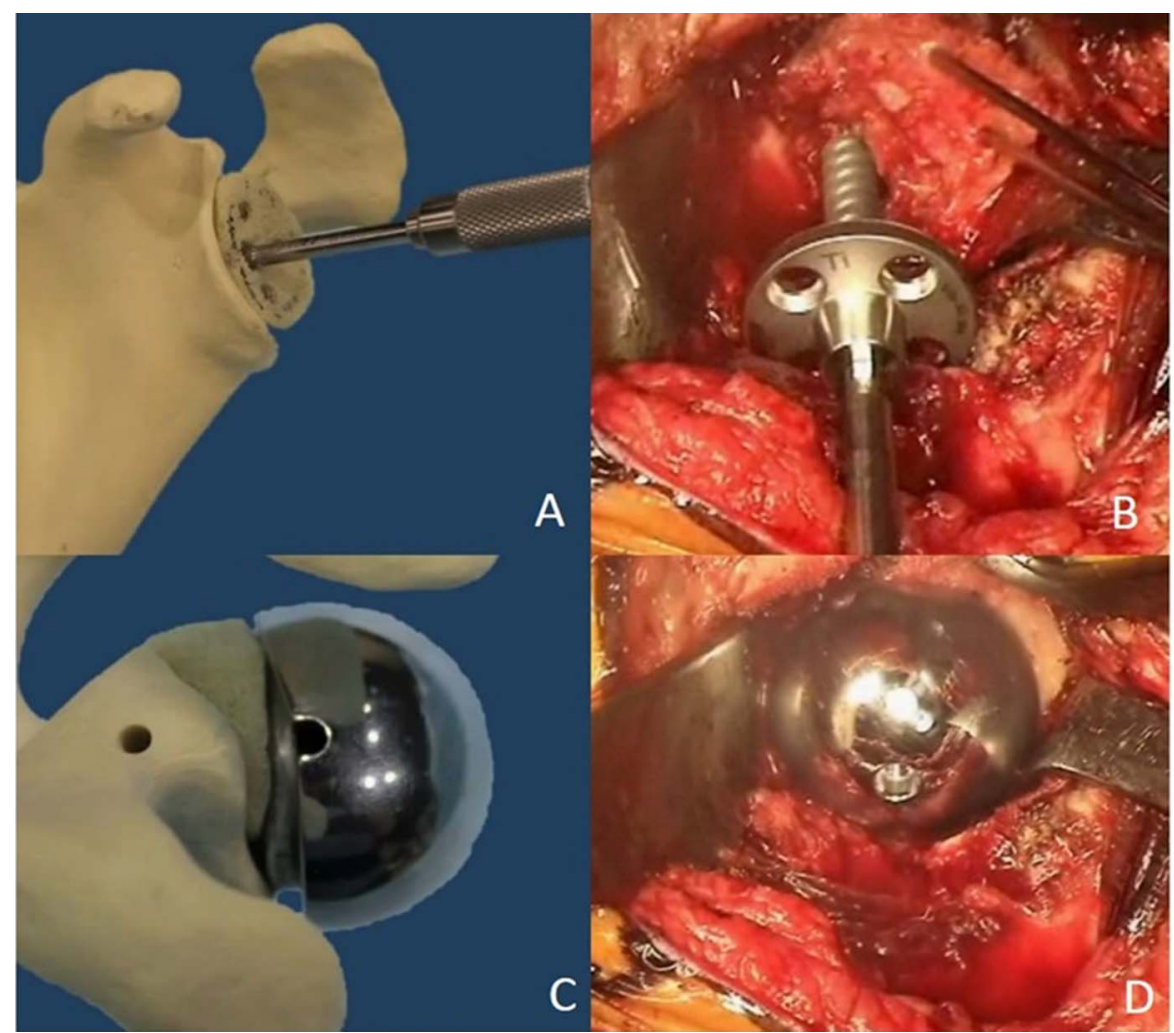

Fig. 1

Figs. 1-A through 1-D Illustrative steps in graft placement and technique. Fig. 1-A A Sawbones graft (Pacific Research Laboratories) shaped to fit the defect with a tap inserted through the graft. Fig. 1-B Intraoperative image of a provisionally pinned humeral head autograft with a screw-in baseplate being inserted to compress the graft into the defect. Fig. 1-C Uncontained defect with a hooded glenosphere oriented to make contact with the graft and load-share with the baseplate. Fig. 1-D Intraoperative image after completed grafting of an uncontained defect and glenosphere contact with the posterior part of the graft.

preoperative values ${ }^{17,18}$. Patient satisfaction was determined on a 10-point scale, with a score of 1 indicating the lowest satisfaction and a score of 10 , the highest satisfaction. Active rangeof-motion measurements were reported, and these were collected preoperatively and postoperatively using a previously described video method ${ }^{19}$. We assessed the postoperative radiographs (anteroposterior, Grashey, scapular Y, and axillary lateral) of each patient at the latest follow-up (mean, 46 months) for evidence of hardware failure; radiolucent lines, indicating loosening; scapular fracture; and bone-graft incorporation. The use of either the conventional or alternative centerline was evaluated on immediate postoperative radiographs for every patient. Complications were identified by both chart and radiographic reviews. Major complications were defined as those resulting in hospital readmission within 90 days of surgery, any readmission requiring further surgery to the shoulder or arm, and any periprosthetic fracture or implant failure that underwent nonoperative treatment because of either patient or surgeon preference.

\section{Surgical Technique}

The senior author (M.A.F.) performed all surgeries using a standard deltopectoral approach. Intraoperatively, in no case was the acceptable threshold percentage (80\%) of contact achieved between the glenoid baseplate (Reverse Shoulder Prosthesis; DJO Surgical) and the host bone. For this reason, the glenoid baseplate was implanted with bone-graft augmentation using the surgical technique previously described by Klein et al. ${ }^{1}$. Selection of graft material was based on intraoperative availability (Fig. 1). Autograft (52 patients; 91\%) was preferentially used when adequate bone could be salvaged from the humeral head, often after humeral head osteotomy (Fig. 2). When allograft (5 patients; 9\%) was required because of insufficient or poorquality autograft bone, a femoral head allograft was used. Glenosphere size was determined on the basis of the soft-tissue tension. The 36- $\mathrm{mm}$ and $40-\mathrm{mm}$ glenosphere sizes (in 40 and 5 patients, respectively) offered a hooded design and could increase contact surface area of the glenosphere with the native scapula, and in many cases this design feature was utilized during surgery. However, 32-mm glenospheres (in 12 patients) were used at the discretion of the senior author in the instances in which it best fit the patient's anatomy.

\section{D-3D Registration}

Image registration for the assessment of native bone support was performed using $3 \mathrm{D}$ reconstruction of preoperative $\mathrm{CT}$ 


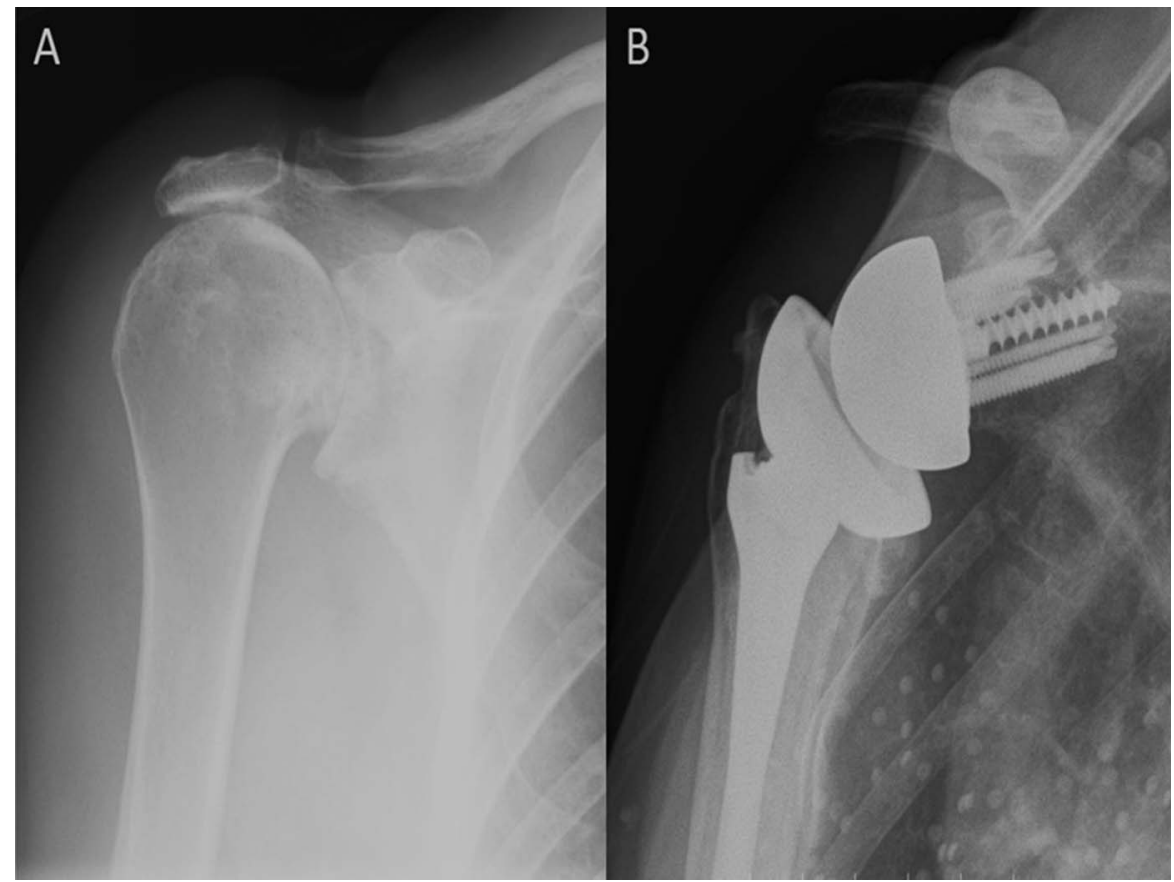

Fig. 2

Figs. 2-A and 2-B A patient who had primary cuff tear arthropathy with substantial superior bone loss. Fig. 2-A Preoperative radiograph. Fig. 2-B Radiograph made 8 years postoperatively showing full incorporation of superior autograft from humeral head.

scan data in combination with postoperative radiographs made 3 months following surgery. Postoperative CT scans were not made, as they are not routinely used in the senior author's practice. Additionally, the goal of the study was to correlate function with the amount of native bone support at the time of implantation irrespective of the amount of bonegraft incorporation over time. The ASES score was used as the surrogate for function in an attempt to correlate native bone support with function. Sizing information for the baseplate and glenosphere was taken from the hospital record, and virtual models of the implants (baseplate and glenosphere) were then created and used for virtual implantation in Mimics 17 (Materialise). The exact position of the implant with respect to the 3D scapular model was determined using contour projection shape-mapping algorithms present in the software, which performs 2D-3D image registration using virtual $\mathrm{x}$-ray emitters in a best-fit shape-matching algorithm as a virtual model is rotated to exactly match the silhouette created by the model on radiographs (Figs. 3 and 4). This shape-matching algorithm has been found to replicate the spatial relationships between anatomy and prosthesis within a mean of $0.60 \pm 0.52 \mathrm{~mm}$ and $1.15^{\circ} \pm 0.87^{\circ 20}$. The acromion was used as a reference, since its shape is not altered by the RSA procedure. However, when the acromion was poorly visualized or fractured, postoperative radiographs were considered inadequate, and these patients were excluded. A total of 6 patients were excluded because of inadequate $\mathrm{CT}$ format, and an additional 7 patients were excluded because of the abovementioned problems with a poorly visualized or fractured acromion on postoperative radiographs. This left 44 patients who had both preoperative CT scans and adequate postoperative radiographs to be included in this arm of the study. The resulting $3 \mathrm{D}$ position of the surgical implant relative to the scapula was then imported into a software program, 3-matic (Materialise), which allowed us to calculate the contact area

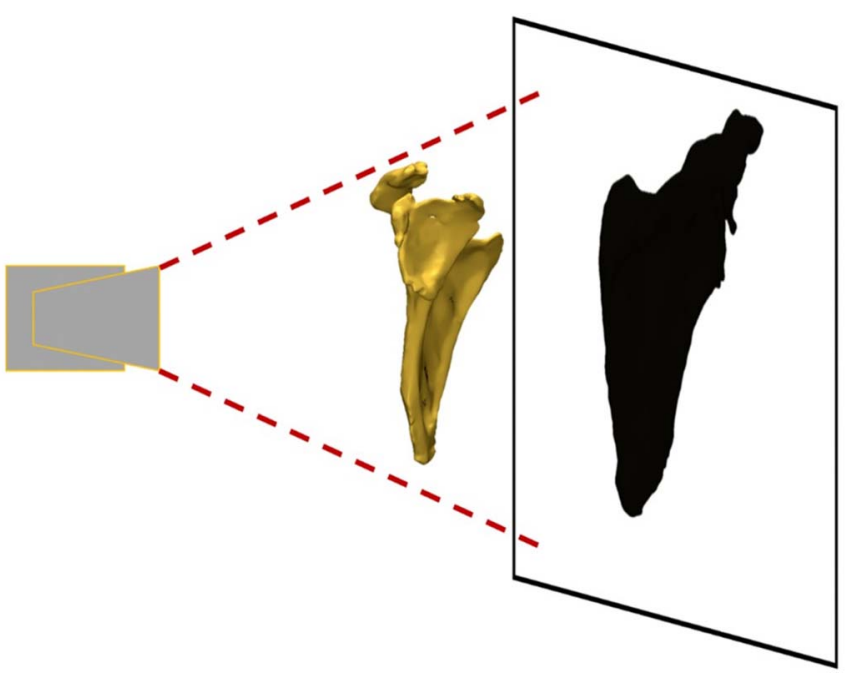

Fig. 3

A simplified example of the virtual emitter system. The light source (left) creates a shadow projection of a virtual model onto a surface (right). Similar to a shadow, the projection is dependent on the location of the model and background relative to the light source. The shadow's outline is representative of the contours that will be fitted. 


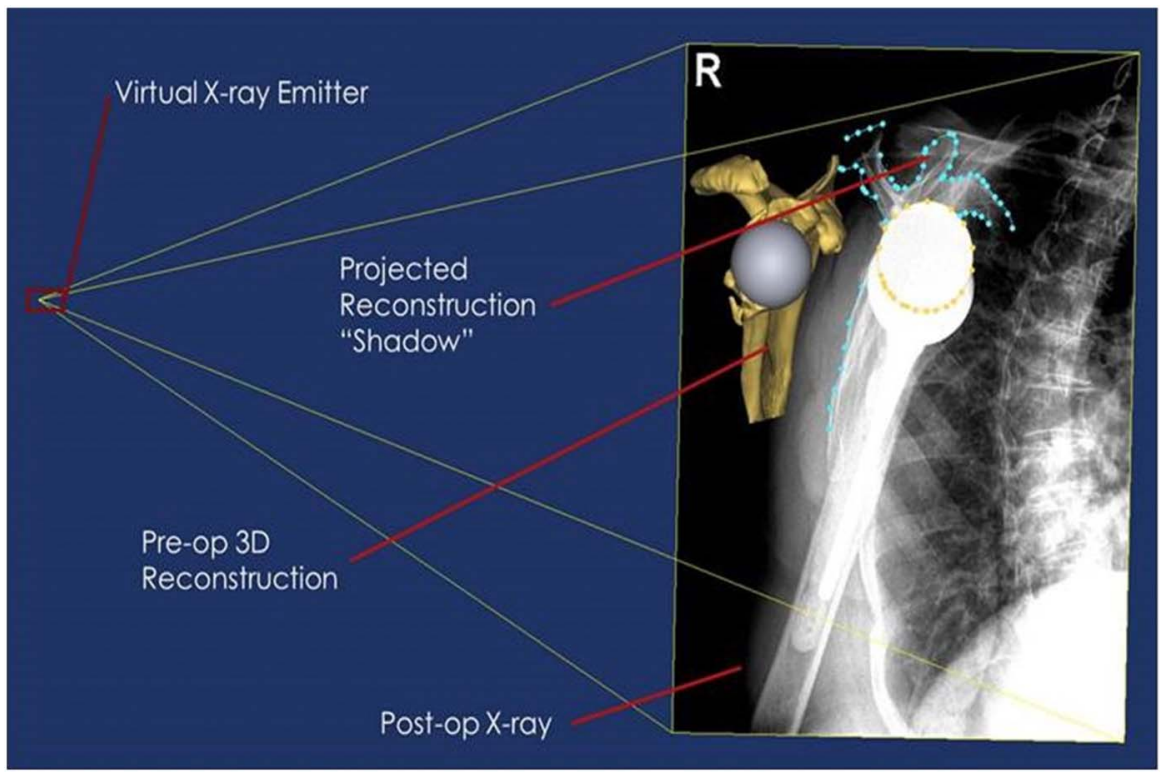

Fig. 4

An example of the virtual workspace. The projected contours are fitted to the user-defined contours, utilizing the 3D models and postoperative radiograph.

of the implant (baseplate and glenosphere) that intersected with the host bone (Fig. 5). This area was normalized as a function of total available surface area and was reported as a percentage.

Application of the registration method applied to the shoulder joint has been validated using a cadaveric model. A fresh-frozen cadaveric shoulder was thawed overnight, and the scapula was disarticulated and dissected of all soft tissues. A CT scan of the scapula was made following parameters identical to those for clinical scans. The senior author created a bone loss model and performed baseplate implantation (Fig. 6). Subsequently, a set of 3 planar radiographs was made. The $2 \mathrm{D}-3 \mathrm{D}$ registration was performed following the methodology described above (Fig. 7). Lastly, the contact area at the baseplate-host bone interface was estimated in the cadaveric model. The baseplate was

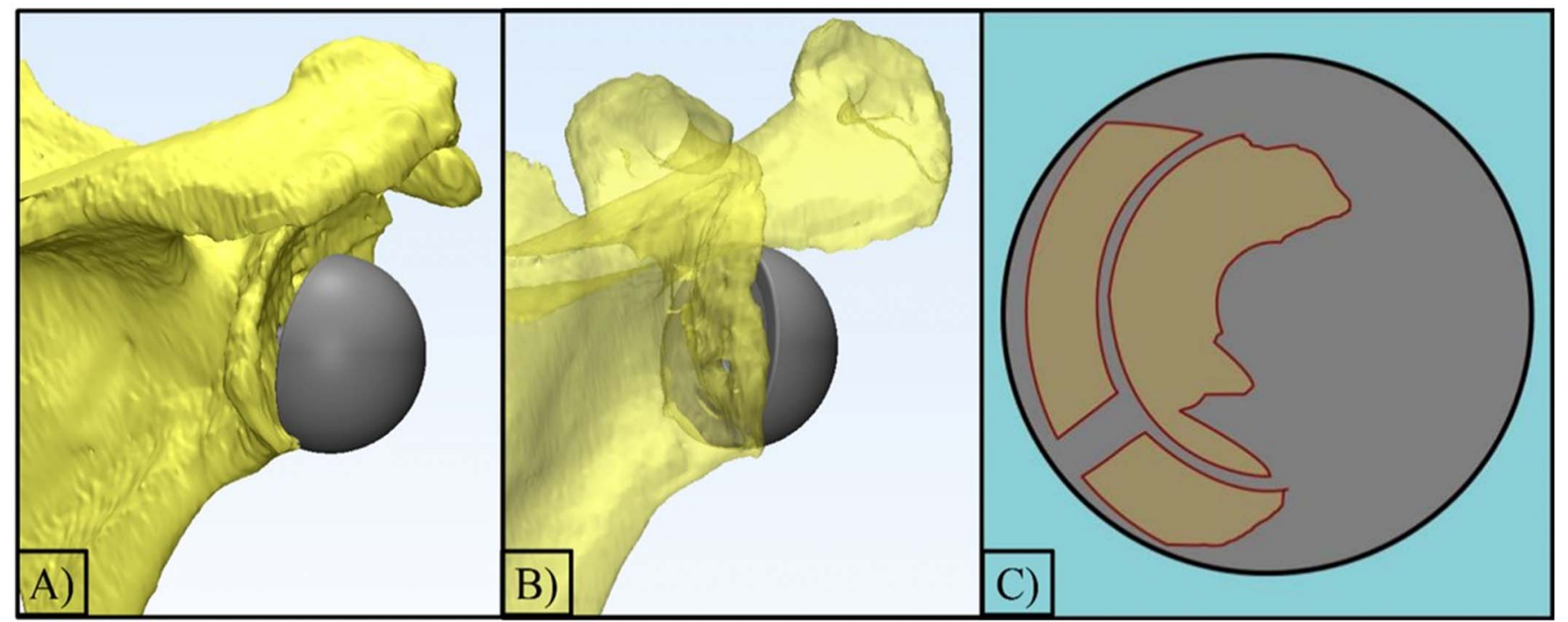

Fig. 5

Figs. 5-A, 5-B, and 5-C An example of the software program, 3-matic (Materialise), that was used to calculate the contact area of the implant (baseplate and glenosphere) that intersected with the host bone. Fig. 5-A The final positioning of the implant on the scapula with a gap between the glenosphere and the host bone. Fig. 5-B The translucent model displays the anterior host bone contact. Fig. 5-C A 2D representation of the intersecting area between the host bone and the baseplate. 

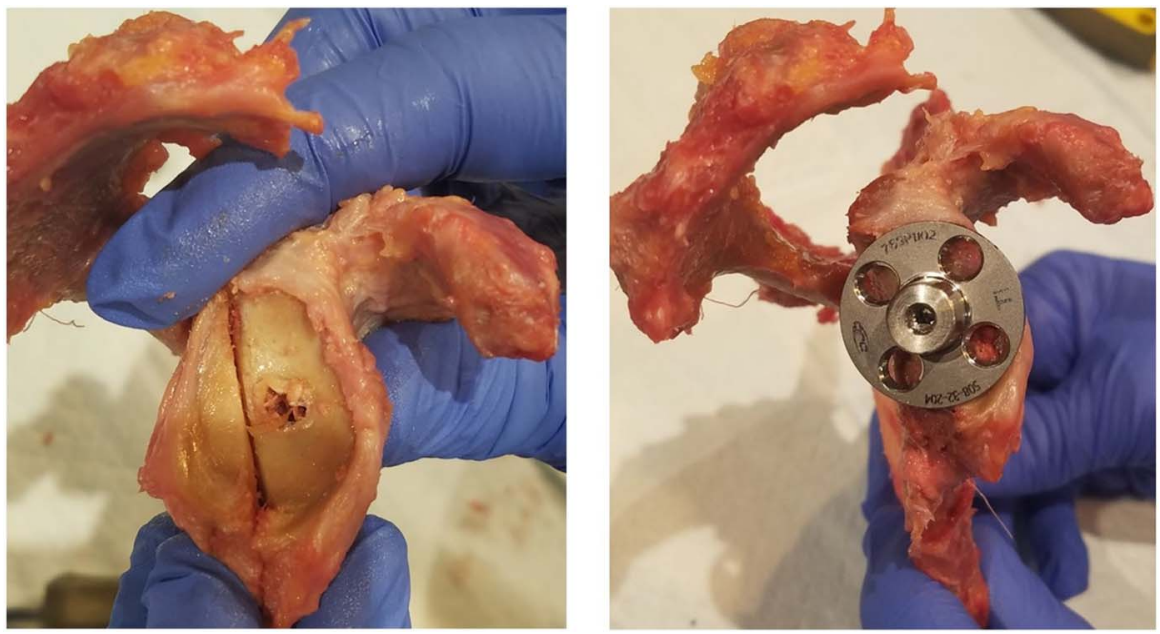

Fig. 6

Bone loss model (left) and the implanted baseplate in the glenoid bone loss model (right).

carefully displaced, and the contact area of the baseplate was covered with a thin layer of acrylic paint and carefully implanted back. After baseplate removal, the area of the glenoid covered with paint was evaluated using digital photography (Fig. 8).

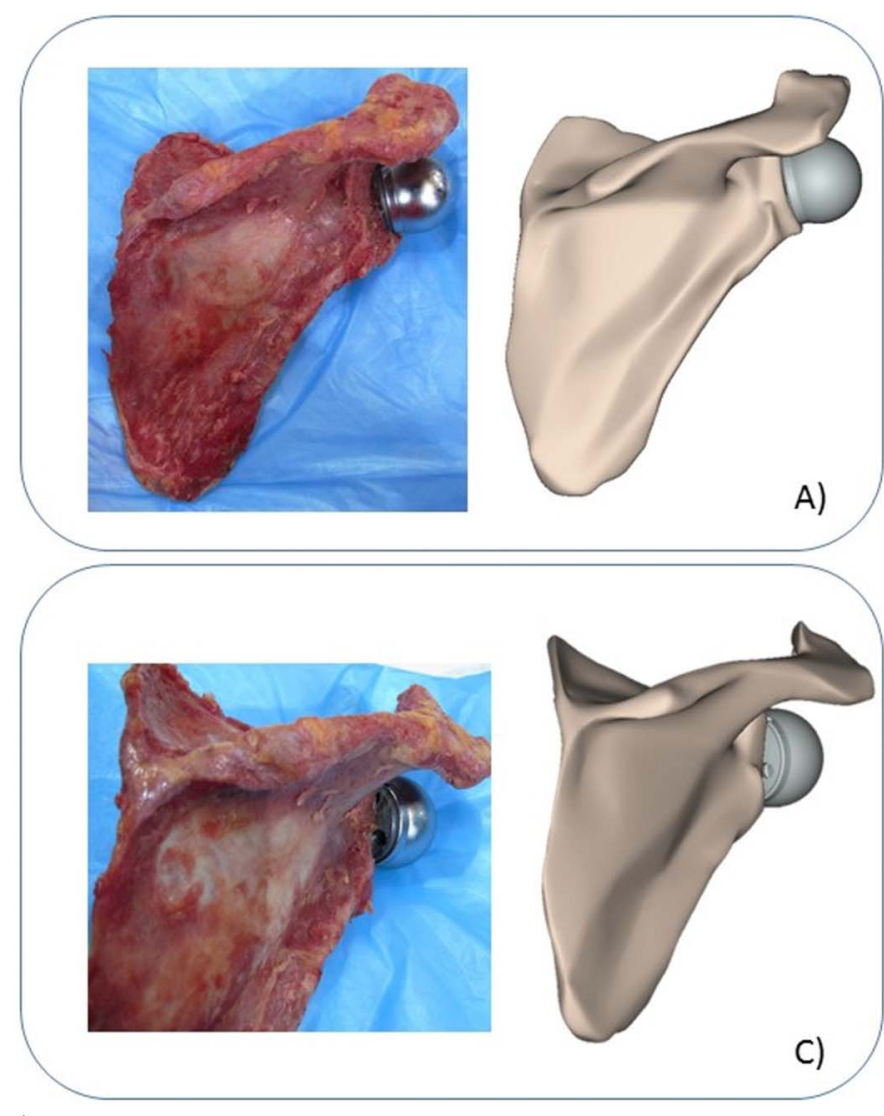

Figs. 7-A through 7-D Comparison of the cadaveric model and the computer model in 4 views. Fig. 7-A Anteroposterior view. Fig. 7-B Posteroanterior view. Fig. 7-C Lateral view. Fig. 7-D Grashey view.

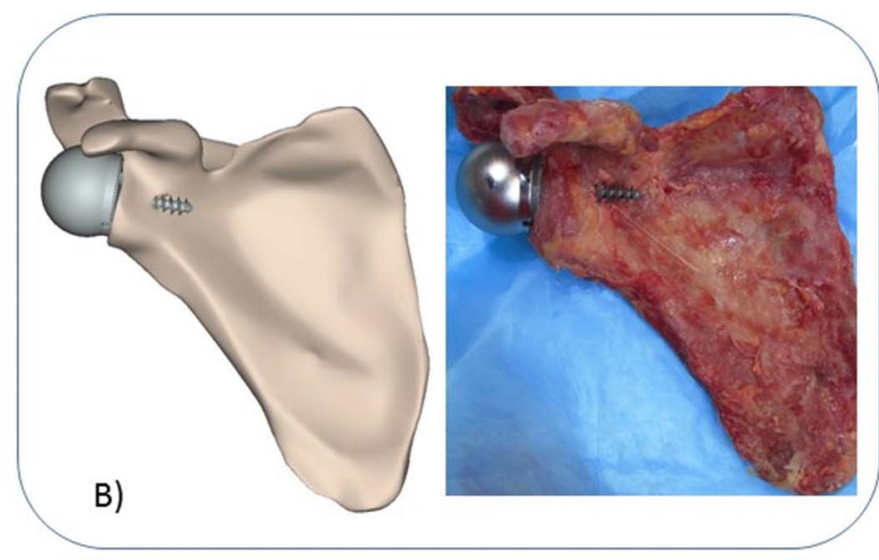

\section{Statistical Analysis}

The normality of all parameters was evaluated (Shapiro-Wilk test). A paired $t$ test or alternative Wilcoxon signed-rank test was used to evaluate outcome measures. Pearson correlation analysis was performed for continuous variables. A p value of

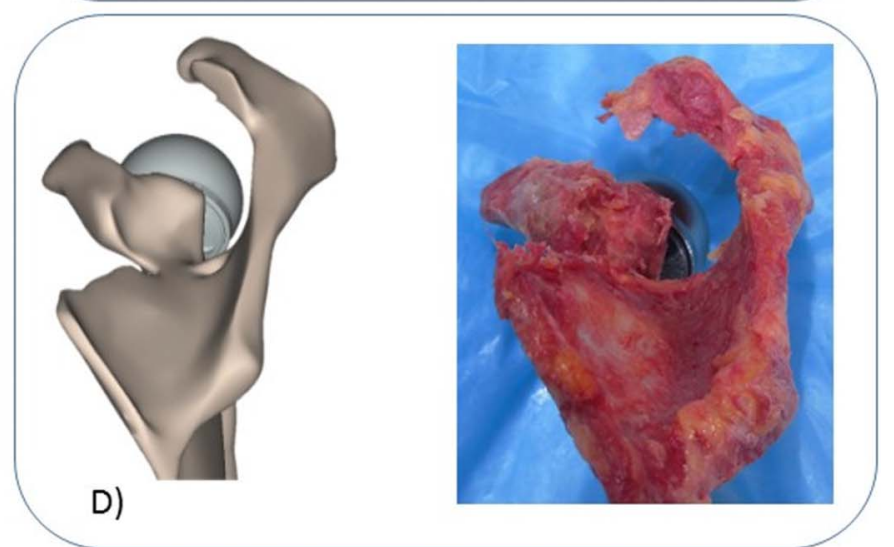

Fig. 7 

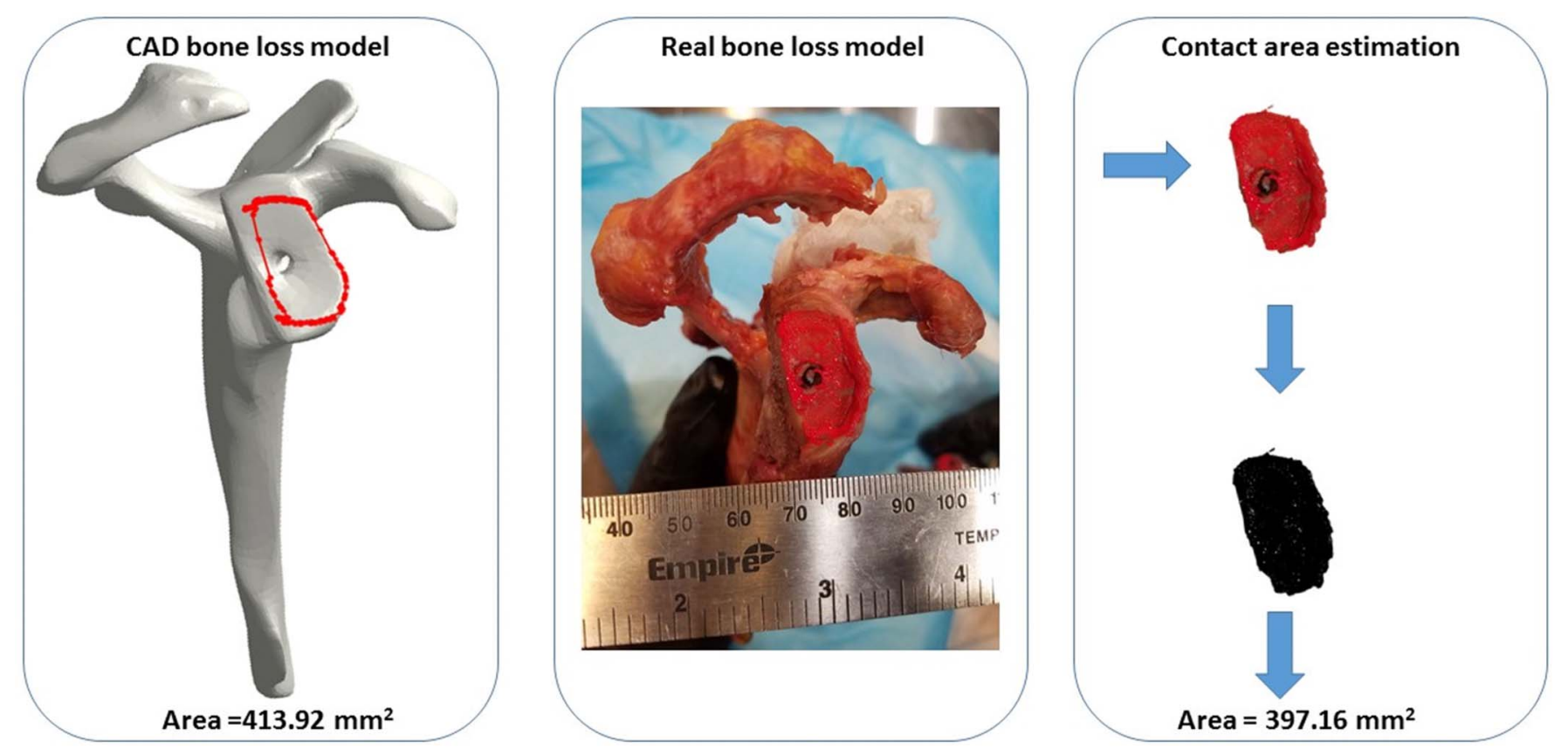

Fig. 8

Estimation of the glenoid-baseplate interface area in the cadaveric model, showing the 2D-3D registration-based area estimation (left), cadaveric model (center), and still picture estimation of the area using image analysis (2D estimation) (right). CAD = computer-aided design.

$<0.05$ was considered significant for all tests, with analyses performed with SPSS software (version 22; IBM).

\section{Results}

verall, the patients with functional outcome measures demonstrated significant improvement from preoperative values (Table III). Patient satisfaction in the study group averaged $8.6 \pm 1.8$ of a possible 10 points. In 56 cases
(98\%), the graft was fully incorporated. There were 4 major complications $(7 \%)$ in the study group, and none of them involved glenoid baseplate failure. One baseplate demonstrated radiolucent lines concerning for loosening; however, the patient did not show signs of clinical failure and therefore did not undergo revision surgery (Grade 4 according to Deutsch et al. ${ }^{21}$ ). Complications required revision surgery in 3 patients. Two shoulders underwent a

\section{TABLE III Preoperative and Postoperative Clinical Outcome Scores and Range of Motion of Primary RSA with Bone Graft}

\begin{tabular}{|c|c|c|c|c|}
\hline & Preop.* & Postop.* & Change & P Value \\
\hline \multicolumn{5}{|l|}{ Outcome scores } \\
\hline ASES function & $17.3 \pm 10.0$ & $33.0 \pm 12.0$ & 15.7 & $<0.0001$ \\
\hline ASES total & $36.7 \pm 16.5$ & $75.3 \pm 18.0$ & 38.6 & $<0.0001$ \\
\hline VAS pain & $6.2 \pm 2.3$ & $1.6 \pm 2.5$ & -4.6 & $<0.0001 \dagger$ \\
\hline \multicolumn{5}{|l|}{ Range of motion } \\
\hline Forward elevation & $69.7^{\circ} \pm 33.8^{\circ}$ & $142.1^{\circ} \pm 42.0^{\circ}$ & $72.4^{\circ}$ & $<0.0001$ \\
\hline Abduction & $64.8^{\circ} \pm 31.8^{\circ}$ & $132.5^{\circ} \pm 41.0^{\circ}$ & $67.7^{\circ}$ & $<0.0001$ \\
\hline External rotation & $29.4^{\circ} \pm 23.5^{\circ}$ & $53.7^{\circ} \pm 40.0^{\circ}$ & $24.3^{\circ}$ & $<0.0001 \dagger$ \\
\hline Internal rotation $キ$ & $2.7 \pm 1.9$ & $4.6 \pm 2.2$ & 1.9 & $0.024 \dagger$ \\
\hline
\end{tabular}


single-stage revision to a long-stemmed implant for humeral loosening, and 1 was treated with open reduction and internal fixation for a periprosthetic fracture. There were 5 acromial or scapular spine fractures $(9 \%)$ noted in the study group. Four patients (7\%) demonstrated scapular notching; 3 had grade 1 and 1 had grade 3, according to the system described by Sirveaux et al. ${ }^{15}$.

Software-reported shape-matching of the prosthesis within our study between projected and generated contours of implants was found to be $\geq 90 \%$. The mean percentage of the implant (baseplate and glenosphere) supported by native bone was $17 \% \pm 12 \%$ (range, $0 \%$ to $50 \%$ ). There was no significant association between host bone coverage and the change in the ASES score $(\mathrm{p}=0.51)$.

Validation of the registration method revealed a good match between computer-based $\left(413.92 \mathrm{~mm}^{2}\right)$ and cadaveric-based $\left(397.16 \mathrm{~mm}^{2}\right)$ estimation of the contact area (Fig. 8).

\section{Discussion}

$\mathbf{R}$ everse shoulder arthroplasty has become a valuable tool Ravailable to shoulder surgeons who treat complex pathology, and in many cases, including primary arthroplasties, the surgeon may encounter severe glenoid bone loss. Most studies of RSA in the setting of glenoid bone loss have focused on revision arthroplasty, and relatively little is known about the results of primary RSA when structural bone graft is needed to restore eroded glenoid bone stock. The results of our study indicate that the use of structural bone graft in primary RSA produces good outcomes that are comparable with outcomes of RSA performed without bonegrafting, and that outcomes are not dependent on the amount of host bone available to provide support under the baseplate $^{22,23}$.

This study represents what we believe is the largest reported series of primary RSAs performed with glenoid bone-grafting for severe glenoid deficiency, and the outcomes in our study group were equivalent to or better than those previously reported ${ }^{8,23,24}$. Neyton et al. reported a series of 9 patients who underwent RSA with glenoid bone-grafting and showed improvement in the pain score and no baseplate loosening; however, poor functional outcomes were reported after 2 years $^{25}$. Levigne et al. studied primary RSA with bone-grafting in 34 patients and found a bone-graft incorporation rate of $72.9 \%$, with improvement in the Constant score and otherwise variable functional outcomes ${ }^{3}$. However, more recently, Jones et al., in a study evaluating a combined cohort of patients managed with primary and revision RSA who underwent structural glenoid grafting, reported improved functional outcomes similar to those in our patients at the latest follow-up ${ }^{23}$. The current study notes a $98 \%$ rate of bone-graft incorporation. The reason for the difference in the outcomes that we report in this series may be related to the substantial experience of the senior author in using RSA to treat a variety of severe pathologies of the shoulder as well as the superior compressive force provided by the implant ${ }^{24}$. The use of a fixed-angle central screw provides for immediate stable fixation by compression of the undersurface of the baseplate against available osseous contact. Proprioception of the increased torque as the undersurface of the baseplate compresses against the osseous contact provides the surgeon immediate feedback on the security of fixation.

Many surgeons now use RSA to treat complex pathology of the shoulder, and use of the device is increasing ${ }^{26}$. Most reports of RSA with bone-grafting have described surgery performed in the revision setting, and it is understood that outcomes of revision surgery are generally poorer and therefore patient and surgeon expectations would be expected to be different ${ }^{13,25,27-29}$. In a large series of revision RSAs performed with bone-grafting (40 patients), Wagner et al. reported that implant survival at 2 and 5 years was $88 \%$ and $76 \%$, respectively $y^{27,28}$. Those authors noted particular concern when a lateralized RSA was implanted, although this effect did not reach significance. The patients in our study all underwent primary RSA with a lateralized glenoid component, and we observed no baseplate failures, with 1 patient at risk of loosening because of radiolucent lines around the baseplate. The amount of host contact between the baseplate and the native glenoid was on average $17 \%$, suggesting that a bone graft to restore a large majority of the glenoid surface was necessary to provide complete coverage of the baseplate. Additionally, Formaini et al. evaluated our screw-in baseplate design biomechanically and determined that a minimum of $50 \%$ bone support was necessary at implantation to keep micromotion below the threshold level to allow for bone ingrowth to support the baseplate ${ }^{30}$. Our method of measuring the actual postoperative placement of the baseplate by utilizing standardized $2 \mathrm{D}$ radiographs and then the preoperative 3D glenoid image to determine the degree of contact with the baseplate provides a method to evaluate implant position after surgical implantation. Despite having a large portion of the implant resting on bone graft in patients with severe glenoid deficiency, there were no glenoidsided failures.

It is important to differentiate our results from those of osseous increased-offset RSAs (BIO-RSAs; Tornier), in which structural bone graft is used to provide increased lateral offset to the glenosphere ${ }^{31,32}$. Bone-grafting for patients in the present study was performed for severe bone loss or deformity that would have otherwise resulted in a lack of support for the glenoid baseplate. The favorable outcomes demonstrated in this cohort could not, in our estimation, have been achieved without the use of structural bone graft. The improvement in all motion parameters, including internal and external rotation, and the low rate of scapular notching also provide evidence that patients with severe glenoid bone loss can anticipate a reliable surgical outcome, with improvement of function and a reduction of pain at an average of 46 months with stable implant interfaces.

Weaknesses of this study relate to its retrospective nature, and to the theoretical limitations imposed by the use of 
modeling software. We also do not routinely make postoperative CT scans to assess for bone graft incorporation, and therefore it is unknown whether this is a factor that influences outcome. However, no baseplate failures were observed in our study cohort, and most patients were greatly improved following surgery to restore function that had been lost because of a severely deformed shoulder.

In conclusion, we found good outcomes in a series of 57 patients who had primary RSA with bone graft augmentation for severe glenoid bone loss. Functional outcome was not related to the degree of native bone support under the glenoid baseplate, making bone-grafting an attractive option in even the most challenging shoulder arthroplasty cases. However, it is important to note that our study population consisted of patients with a great variation in the types of glenoid bone loss, and our analysis was not sufficiently powered to evaluate this variation. To our knowledge, the present study is the largest reported series of patients undergoing primary RSA with bonegrafting for severe glenoid bone deficiency. On the basis of the results reported in this series, we continue to perform primary RSA with bone graft augmentation for severe glenoid bone loss, and we counsel patients that outcomes can be excellent despite their severe glenoid bone loss.

Adam Lorenzetti, $\mathrm{MD}^{1}$

Jonathan J. Streit, $\mathrm{MD}^{1}$

Andres F. Cabezas, $\mathrm{MS}^{2}$

Kaitlyn N. Christmas, BS ${ }^{2}$

Joey LaMartina II, $\mathrm{MD}^{1}$

Peter Simon, $\mathrm{PhD}^{2}$

Mark A. Frankle, $\mathrm{MD}^{1}$

${ }^{1}$ Shoulder and Elbow Service, Florida Orthopaedic Institute, Tampa, Florida

${ }^{2}$ Clinical Research, Foundation for Orthopaedic Research and Education, Tampa, Florida

E-mail address for M.A. Frankle: mfrankle@floridaortho.com

ORCID iD for M.A. Frankle: 0000-0001-6427-0665

\section{References}

1. Klein SM, Dunning $P$, Mulieri $P$, Pupello D, Downes K, Frankle MA. Effects of acquired glenoid bone defects on surgical technique and clinical outcomes in reverse shoulder arthroplasty. J Bone Joint Surg Am. 2010 May;92(5):1144-54.

2. Klika BJ, Wooten CW, Sperling JW, Steinmann SP, Schleck CD, Harmsen WS, Cofield RH. Structural bone grafting for glenoid deficiency in primary total shoulder arthroplasty. J Shoulder Elbow Surg. 2014 Jul;23(7):1066-72. Epub 2013 Dec 14.

3. Levigne C, Garret J, Grosclaude S. Results of glenoid bone graft in primary reverse shoulder arthroplasty. In: Walch G, Boileau P, Molé D, Favard L, Lévigne C, Sirveaux F, editors. Shoulder concepts 2010: the glenoid. Montpellier: Sauramps Medical; 2010. p 403-9.

4. Levy J, Frankle M, Mighell M, Pupello D. The use of the reverse shoulder prosthesis for the treatment of failed hemiarthroplasty for proximal humeral fracture. $J$ Bone Joint Surg Am. 2007 Feb;89(2):292-300.

5. Hill JM, Norris TR. Long-term results of total shoulder arthroplasty following bone-grafting of the glenoid. J Bone Joint Surg Am. 2001 Jun;83(6):877-83.

6. Ho JC, Sabesan VJ, lannotti JP. Glenoid component retroversion is associated with osteolysis. J Bone Joint Surg Am. 2013 Jun 19;95(12):e82.

7. Holcomb JO, Cuff D, Petersen SA, Pupello DR, Frankle MA. Revision reverse shoulder arthroplasty for glenoid baseplate failure after primary reverse shoulder arthroplasty. J Shoulder Elbow Surg. 2009 Sep-Oct;18(5):717-23. Epub 2009 Mar 17.

8. Scalise JJ, lannotti JP. Bone grafting severe glenoid defects in revision shoulder arthroplasty. Clin Orthop Relat Res. 2008 Jan;466(1):139-45. Epub 2008 Jan 3.

9. Boileau P, Watkinson D, Hatzidakis AM, Hovorka I. Neer Award 2005: the Grammont reverse shoulder prosthesis: results in cuff tear arthritis, fracture sequelae, and revision arthroplasty. J Shoulder Elbow Surg. 2006 Sep-Oct;15(5):527-40. 10. Chacon A, Virani N, Shannon R, Levy JC, Pupello D, Frankle M. Revision arthroplasty with use of a reverse shoulder prosthesis-allograft composite. J Bone Joint Surg Am. 2009 Jan;91(1):119-27.

11. Melis $B$, Bonnevialle N, Neyton $L$, Lévigne $C$, Favard L, Walch G, Boileau $P$. Glenoid loosening and failure in anatomical total shoulder arthroplasty: is revision with a reverse shoulder arthroplasty a reliable option? J Shoulder Elbow Surg. 2012 Mar;21(3):342-9. Epub 2011 Nov 1.

12. Patel DN, Young B, Onyekwelu I, Zuckerman JD, Kwon YW. Reverse total shoulder arthroplasty for failed shoulder arthroplasty. J Shoulder Elbow Surg. 2012 Nov;21(11):1478-83. Epub 2012 Feb 22.

13. Saltzman BM, Chalmers PN, Gupta AK, Romeo AA, Nicholson GP. Complication rates comparing primary with revision reverse total shoulder arthroplasty. J Shoulder Elbow Surg. 2014 Nov;23(11):1647-54. Epub 2014 Jun 28.

14. Walker M, Willis MP, Brooks JP, Pupello D, Mulieri PJ, Frankle MA. The use of the reverse shoulder arthroplasty for treatment of failed total shoulder arthroplasty. J Shoulder Elbow Surg. 2012 Apr;21(4):514-22. Epub 2011 Jun 8.
15. Sirveaux F, Favard L, Oudet D, Huquet D, Walch G, Molé D. Grammont inverted total shoulder arthroplasty in the treatment of glenohumeral osteoarthritis with massive rupture of the cuff. Results of a multicentre study of 80 shoulders. J Bone Joint Surg Br. 2004 Apr;86(3):388-95.

16. Walch G, Badet R, Boulahia A, Khoury A. Morphologic study of the glenoid in primary glenohumeral osteoarthritis. J Arthroplasty. 1999 Sep;14(6):756-60. 17. Kocher MS, Horan MP, Briggs KK, Richardson TR, O'Holleran J, Hawkins RJ. Reliability, validity, and responsiveness of the American Shoulder and Elbow Surgeons subjective shoulder scale in patients with shoulder instability, rotator cuff disease, and glenohumeral arthritis. J Bone Joint Surg Am. 2005 Sep;87(9):2006-11.

18. Godfrey J, Hamman R, Lowenstein S, Briggs K, Kocher M. Reliability, validity, and responsiveness of the Simple Shoulder Test: psychometric properties by age and injury type. J Shoulder Elbow Surg. 2007 May-Jun;16(3):260-7. Epub 2006 Dec 22.

19. Cuff D, Pupello D, Virani N, Levy J, Frankle M. Reverse shoulder arthroplasty for the treatment of rotator cuff deficiency. J Bone Joint Surg Am. 2008 Jun;90 (6):1244-51.

20. Vigneron L, Delport H, De Boot S. Accuracy assessment of $2 D$ X-ray to $3 D C T$ registration for measuring 3D postoperative implant position [white paper]. 2014 http://www.materialise.com/en/resources/white-papers/accuracy-assessmentof-2d-x-ray-to-3d-ct-registration-for-measuring-3d. Accessed 2017 June 6.

21. Deutsch A, Abboud JA, Kelly J, Mody M, Norris T, Ramsey ML, lannotti JP, Williams GR. Clinical results of revision shoulder arthroplasty for glenoid component loosening. J Shoulder Elbow Surg. 2007 Nov-Dec;16(6):706-16. Epub 2007 Oct 10. 22. Kiet TK, Feeley BT, Naimark M, Gajiu T, Hall SL, Chung TT, Ma CB. Outcomes after shoulder replacement: comparison between reverse and anatomic total shoulder arthroplasty. J Shoulder Elbow Surg. 2015 Feb;24(2):179-85. Epub 2014 Sep 9.

23. Jones RB, Wright TW, Zuckerman JD. Reverse total shoulder arthroplasty with structural bone grafting of large glenoid defects. J Shoulder Elbow Surg. 2016 Sep;25(9):1425-32. Epub 2016 Mar 31.

24. Frankle M. Rotator cuff deficiency of the shoulder. In: Frankle MA, Virani N, Pupello D, Gutierrez S, editors. Rationale and biomechanics of the reverse shoulder prosthesis: the American experience. New York: Thieme; 2006. p 10-21.

25. Neyton L, Boileau P, Nové-Josserand L, Edwards TB, Walch G. Glenoid bone grafting with a reverse design prosthesis. J Shoulder Elbow Surg. 2007 May-Jun;16 (3)(Suppl):S71-8. Epub 2006 Sep 20.

26. Jain NB, Yamaguchi K. The contribution of reverse shoulder arthroplasty to utilization of primary shoulder arthroplasty. J Shoulder Elbow Surg. 2014 Dec;23 (12):1905-12. Epub 2014 Oct 7.

27. Wagner E, Houdek MT, Griffith T, Elhassan BT, Sanchez-Sotelo J, Sperling JW, Cofield RH. Glenoid bone-grafting in revision to a reverse total shoulder arthroplasty. J Bone Joint Surg Am. 2015 Oct 21;97(20):1653-60. 
28. Tashjian R. No bone? No problem! Is bone-grafting at the time of revision to a reverse shoulder arthroplasty a reasonable option? Commentary on an article by Eric Wagner, MD, et al.: "Glenoid bone-grafting in revision to a reverse total shoulder arthroplasty". J Bone Joint Surg Am. 2015 Oct 21;97(20):e68.

29. Werner BS, Böhm D, Abdelkawi A, Gohlke F. Glenoid bone grafting in reverse shoulder arthroplasty for long-standing anterior shoulder dislocation. J Shoulder Elbow Surg. 2014 Nov;23(11):1655-61. Epub 2014 May 14.

30. Formaini NT, Everding NG, Levy JC, Santoni BG, Nayak AN, Wilson C, Cabezas AF. The effect of glenoid bone loss on reverse shoulder arthroplasty baseplate fixation. J Shoulder Elbow Surg. 2015 Nov;24(11):e312-9. Epub 2015 Jul 9.

31. Athwal GS, MacDermid JC, Reddy KM, Marsh JP, Faber KJ, Drosdowech D. Does bony increased-offset reverse shoulder arthroplasty decrease scapular notching? J Shoulder Elbow Surg. 2015 Mar;24(3):468-73. Epub 2014 Oct 22.

32. Boileau $P$, O'Shea K, Moineau G, Roussane Y. Bony increased-offset reverse shoulder arthroplasty (BIO-RSA) for cuff tear arthropathy. Oper Tech Orthop. 2011;21(1):69-78. 